\title{
Exploration of the main sites for the transformation of normal prion protein (PrPC) into pathogenic prion protein (PrPsc)
}

\author{
Xi-Lin Liu ${ }^{1}$, Xiao-Li Feng ${ }^{1,2}$, Guang-Ming Wang ${ }^{1}$, Bin-Bin Gong ${ }^{1}$, \\ Waqas Ahmad ${ }^{1,3}$, Nan-Nan Liu ${ }^{1}$, Yuan-Yuan Zhang ${ }^{1}$, \\ Li Yang ${ }^{1}$, Hong-Lin Ren ${ }^{1}$, Shu-Sen Cui ${ }^{1}$ \\ ${ }^{1}$ China-Japan Union Hospital of Jilin University, Key Laboratory of Zoonosis Research, \\ Ministry of Education/Institute of Zoonosis, Jilin University, Changchun 130062, China \\ ${ }^{2}$ Biological safety protection third-level laboratory, Kunming Institute of Zoology, \\ Chinese Academy of Sciences, Kunming 650223, China \\ ${ }^{3}$ Section of Epidemiology and Public Health, College of Veterinary and Animal Sciences, Jhang 35200, Pakistan \\ sscui916@126.com; renhl@jlu.edu.cn
}

Received: September 13, 2016 Accepted: March 10, 2017

\begin{abstract}
Introduction: The functions and mechanisms of prion proteins $\left(\mathrm{PrP}^{\mathrm{C}}\right)$ are currently unknown, but most experts believe that deformed or pathogenic prion proteins $\left(\mathrm{PrP}^{\mathrm{Sc}}\right)$ originate from $\operatorname{PrP}^{\mathrm{C}}$, and that there may be plural main sites for the conversion of normal $\operatorname{PrP}^{\mathrm{C}}$ into $\mathrm{PrP}^{\mathrm{Sc}}$. In order to better understand the mechanism of $\operatorname{PrP}^{\mathrm{C}}$ transformation to $\operatorname{PrP}^{\mathrm{Sc}}$, the most important step is to determine the replacement or substitution site. Material and Methods: BALB/c mice were challenged with prion RML strain and from 90 days post-challenge ( $\mathrm{dpc}$ ) mice were sacrificed weekly until all of them had been at $160 \mathrm{dpc}$. The ultra-structure and pathological changes of the brain of experimental mice were observed and recorded by transmission electron microscopy. Results: There were a large number of pathogen-like particles aggregated in the myelin sheath of the brain nerves, followed by delamination, hyperplasia, swelling, disintegration, phagocytic vacuolation, and other pathological lesions in the myelin sheath. The aggregated particles did not overflow from the myelin in unstained samples. The phenomenon of particle aggregation persisted all through the disease course, and was the earliest observed pathological change. Conclusion: It was deduced that the myelin sheath and lipid rafts in brain nerves, including axons and dendrites, were the main sites for the conversion of $\operatorname{PrP}^{\mathrm{C}}$ to $\operatorname{PrP}^{\mathrm{Sc}}$, and the $\operatorname{PrP}^{\mathrm{Sc}}$ should be formed directly by the conversion of protein conformation without the involvement of nucleic acids.
\end{abstract}

Keywords: mice, brain, prion proteins, myelin sheath, particle aggregation.

\section{Introduction}

Prion diseases predominantly affect the nervous system leading to fatal and untreatable degenerative disorders of the brain, which are called transmissible spongiform encephalopathies (TSEs). The causative agent of TSEs has been identified as a prion known to be an abnormal isoform (Scrapie prion protein, $\mathrm{PrP}^{\mathrm{Sc}}$ ) of a normal cellular prion protein $\left(\mathrm{PrP}^{\mathrm{C}}\right)(5) . \mathrm{PrP}^{\mathrm{C}}$ is found in all cells of the body, with relatively high levels in the brain. $\mathrm{PrP}^{\mathrm{Sc}}$ is able to induce transformation of the normal prion protein nearby into the misfolded and infectious prion (23).

$\mathrm{PrP}^{\mathrm{Sc}}$ differs from $\mathrm{PrP}^{\mathrm{C}}$ neither in its covalent structure nor post-translational modification, but only in its conformation. $\mathrm{PrP}^{\mathrm{Sc}}$ mainly has $\alpha$-sheeted (helicalor $\beta$-sheet) conformation, while $\operatorname{PrP}^{\mathrm{C}}$ chiefly shows helical conformation (6). A substantial proportion of investigators believe that $\operatorname{PrP}^{\mathrm{Sc}}$ (an abnormal conformer) can convert $\operatorname{PrP}^{\mathrm{C}}$ (the normal cellular protein) into $\mathrm{PrP}^{\mathrm{Sc}}$. This transformation process may be accomplished through $\operatorname{PrP}^{\mathrm{Sc}}$ serving either as a template or a seed to induce transformation of $\operatorname{PrP}^{\mathrm{C}}$ (4). Hence, prion diseases belong to a wide group of conformational disorders or diseases of protein misfolding. The prion theory is almost universally accepted nowadays and its author has been honoured by the Nobel prize (31), although the theory remains to be formally proved. Great success has been achieved in research, yet it is still difficult to explain the reason for variation in scrapie (10). 
Furthermore, there are various clarification theories pertaining to the mechanism behind how and where $\mathrm{PrP}^{\mathrm{Sc}}$ induces the transformation of $\mathrm{PrP}^{\mathrm{C}}$. Some pathological phenomena caused by prion diseases like vacuolisation and myelin sheath puff region changes may at least in part originate from $\mathrm{PrP}^{\mathrm{Sc}}$ accumulation (34), and several studies have speculated that myelin sheath cytoplasmic membrane/sterol rafts of nerve fibres and endoplasmic reticulum may be the main conversion sites of $\operatorname{PrP}^{\mathrm{C}}$ into $\mathrm{PrP}^{\mathrm{Sc}}$. Irrespective of whether there is or is not an association with the $\operatorname{PrP}^{\mathrm{Sc}}$ accumulations, it is still important to clarify early pathogenetic phenomena. The mechanism of the conformation conversion of prion also involves relative special environments, various relative elements, and predisposition factors (25). Nonetheless, how the exogenous $\mathrm{PrP}^{\mathrm{Sc}}$ enters the brain and central nervous system along the nerve after the infection of the peripheral nervous system with prion is still a mystery. In general, it is believed that the prion protein passes through a nerve fibre. However, the particular transformation mechanism is not clear $(3,18,28) . \operatorname{PrP}^{\mathrm{C}}$ is attached to the exterior of the plasma membrane by its glycosyl-phosphatidyl-inositol (GPI) anchor. Currently, a large number of ligands are known to interact with $\operatorname{PrP}^{\mathrm{C}}$ that have also been implicated in a wide range of cellular functions. The way in which $\operatorname{PrP}^{\mathrm{C}}$ is anchored to the membrane plays an important role in turnover and pathogenesis of $\mathrm{PrP}^{\mathrm{Sc}}$. GPI anchors do not extend beyond the top leaflet of the lipid bilayer. Therefore, the proteins such as $\mathrm{PrP}^{\mathrm{C}}$ must be associated with proteins containing transmembrane sections for them to be able to communicate in the intercellular processes. Current data suggest that the clustering of $\operatorname{PrP}^{\mathrm{C}}$ into distinct membrane patches may be due to the association of $\operatorname{PrP}^{\mathrm{C}}$ with transmembrane proteins responsible for trafficking $\mathrm{PrP}^{\mathrm{C}}$ into endocytic pathways (15). Moreover, involvement of transmembrane proteins in the pathological conversion of $\mathrm{PrP}^{\mathrm{C}}$ into $\mathrm{PrP}^{\mathrm{Sc}}$ remains unknown.

In this study, we observed the ultra-structure and pathological changes of the brain of mice infected with prion strain RML to define the mechanism of conformation changes of prion protein, the substitution sites, and the conditions for the transformation of the prion protein into the pathogenic form. The lesions in myelin sheath of nerve fibres including dendrites and axons were investigated to know how $\operatorname{PrP}^{\mathrm{C}}$ converts into $\mathrm{PrP}^{\mathrm{Sc}}$ at the main substitution sites of $\operatorname{PrP}^{\mathrm{C}}$.

\section{Material and Methods}

Prion strain source and experimental animals. The prion strain RML was kindly provided by the China Animal Health \& Epidemiology Center, National Foreign Animal Disease Diagnostic Center (Qingdao). Female BALB/c mice (about $21 \mathrm{~g}$ b.w.) were raised in cages with occupancy of five and abundant supply of feed and water at a temperature of $22^{\circ} \mathrm{C} \pm 2^{\circ} \mathrm{C}$ and relative humidity of $55 \% \pm 5 \%$ under natural daylight conditions.

Groups and treatment of animals. There were 70 mice divided into four groups. The test group consisted of 55 mice challenged with mouse-adapted prion strain RML. The three control groups included a blank control (five mice) treated with physiological saline $(0.85 \%$ $\mathrm{NaCl}$ ), an experimental control (five mice) with brain homogenates of normal mice, and a normal control (five mice) without any treatment. The brain homogenates of mice infected with prion strain RML were prepared as mouse-adapted prion strain RML, which was diluted 10fold with physiological saline as prion challenge solution. After anaesthesia, $25 \mu \mathrm{L}$ of prion challenge solution was injected into the brains of mice in the test group. Meanwhile, mice in the blank control group and experimental control group were respectively injected with the same amount of physiological saline or brain homogenates of normal mice by the same injection method. Changes in the behaviour and body weight of the mice were regularly observed and recorded.

Sampling of mouse brain. From $90 \mathrm{dpc}$ (when the primal nervous symptoms start to appear), the brains of five mice in the test group were sampled once a week and immediately fixed in $2.5 \%$ glutaraldehyde buffer (0.1 M sodium phosphate, $\mathrm{pH}$ 7.4) for ultra-structure observation. Brain samples from the five mice in each of the control groups were collected at $160 \mathrm{dpc}$, and handled similarly to the test group.

Ultra-structure observation of mouse brain under transmission electron microscope (TEM). Preparation of pathological slices from samples of fixed mouse brain was carried out according to previous reports $(13,16)$. In brief, about $2 \mathrm{~mm}^{3}$ of brain samples for TEM were immersed in $2.5 \%$ glutaraldehyde buffer (pH 7.4 with $0.1 \mathrm{M}$ sodium phosphate) and kept at $4^{\circ} \mathrm{C}$ for more than $24 \mathrm{~h}$, and then washed three times in cold sodium phosphate buffer. Later, the brain tissues were post-fixed in $1 \%$ osmium tetroxide solution for $2 \mathrm{~h}$ at $4^{\circ} \mathrm{C}$. Following that, the fixed tissues were dehydrated on an increasing step ethanol gradient, sunk into propylene oxide for $60 \mathrm{~min}$, and embedded in SPI-PON812 kit (SPI Supplies, USA) for $12 \mathrm{~h}$ according to the instructions of manufacturer. Ultrathin sections were cut using a Leica EM UC7 ultramicrotome (Leica, Germany) and stained with uranyl acetate and lead citrate. Finally, the ultrastructure of the brains was observed with a Hitachi H-7650 TEM (Hitachi, Japan) at $80 \mathrm{kV}$.

\section{Results}

Observation of mental status and behaviour. The mice started to present clinical symptoms from 90 days after RML challenge, and the main symptoms were depression, autism, loss of hair lustre, and ataxia. With the progress of the infection, lethargy and sensory disturbance appeared in the late period. 
Observation of myelinated nerves in the control groups. As shown in Fig. 1A, the structures of myelinated nerves from the normal control group at $160 \mathrm{dpc}$ were compact, the myelin membrane and myelin were closely connected without separation, and there was no stratification in the myelin. Myelinated vacuoles could be seen occasionally and the subcellular structures like mitochondria were integrated in the sheath. Structural abnormality of myelinated nerves appeared neither in the blank control group (Fig. 1B) nor in the experimental control group (Fig. 1C) at 160 dpc just as it did not in the normal control.

Observation of myelinated nerves in the test group from 90 to 160 dpc. Changes of myelin were shown at $90 \mathrm{dpc}$. The vacuolar separation had become larger in myelin and increased gradually, more and more caveolae had emerged on the surface of myelin sheaths with tension reduction, and stratification and separation between the cytoplasmic membrane and myelin had also begun to take place. In addition, deposition of dispersed black particles (uncertain pathogens) had increased, and become very small. The myelin had begun to deform and the structure of myelin sheaths was fuzzy. These changes were obvious especially between the two nerve fibres (Fig. 2A). As seen in Fig. 2B, it was also found that local structure of myelin had become blurred and disintegrated, and the original inherent structure of myelin had almost disappeared. Some of the black depositions had accumulated into larger particles. Furthermore, the detached myelin plasmalemma was shrunken and detached vacuoles were also observed. However, the intracellular structure including organelles had not changed obviously, and only parts of the cytoplasmic membrane and myelin sheath were detached.

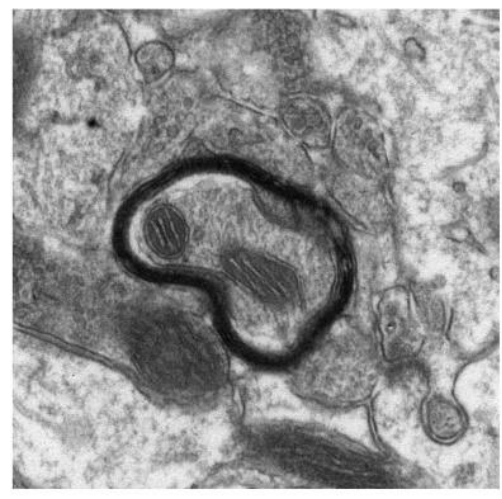

A. The normal control $(10000 \times)$

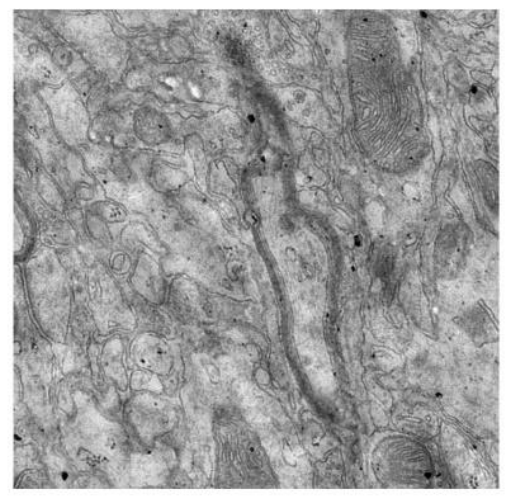

B. The blank control $(5000 \times)$

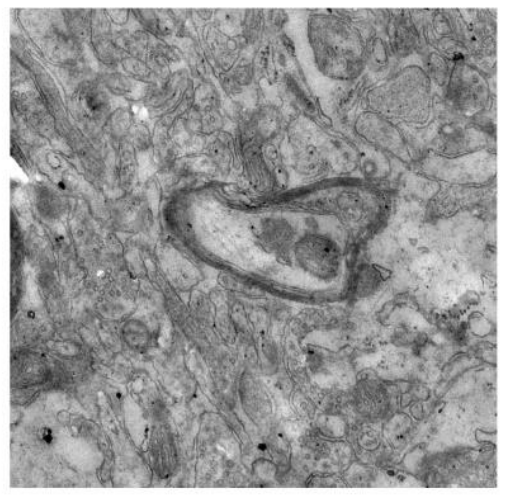

C. The experimental control $(5000 \times)$

Fig. 1. The myelinated nerves of mouse brain from three control groups including the normal control (A), the blank control (B), and the experimental control (C). The normal control received no treatment, the blank control was treated with physiological saline $(0.85 \%$ $\mathrm{NaCl}$ ), and the experimental control was treated with brain homogenates of normal mice. The myelin sheaths in these control groups were compact without stratification in the myelin, and subcellular structures like mitochondria were integrated

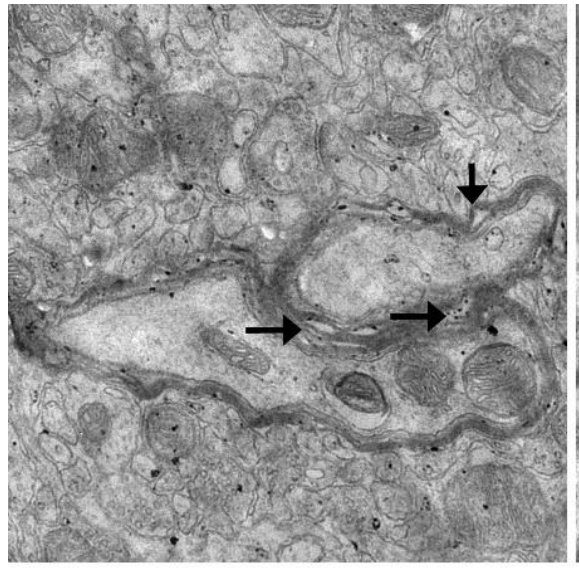

A. $5000 \times$

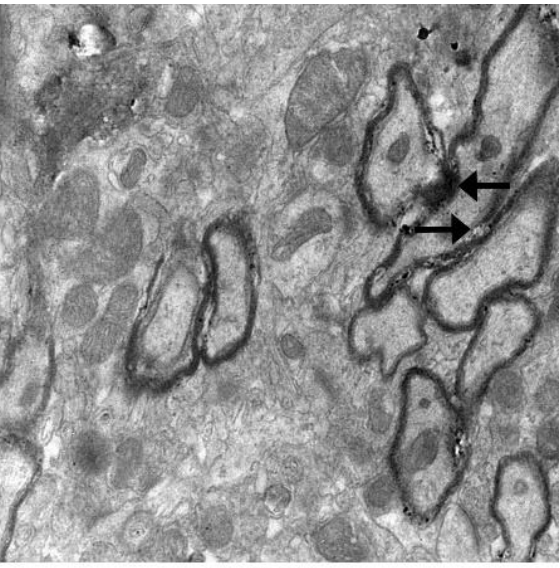

B. $3000 \times$

Fig. 2. Myelinated nerves of mouse brain from the test group at 90 days post challenge. Rightward arrows indicate structures of myelin sheath between two nerve fibres which had become fuzzy and deformed, a leftward arrow indicates that there were some black depositions accumulated into larger particles, and a downward arrow indicates the detached invaginated myelin plasmalemma and vacuoles 
At $97 \mathrm{dpc}$, the myelin had become curved with tension markedly changed to looseness and there was apparent stratification in the myelin, clear deposition or polymerisation of dispersed particles, and an unintegrated cytoplasmic membrane (Fig. 3A). The structure of mitochondria was almost intact, but some parts of the mitochondrial membrane had broken down, and were tending toward degeneration. The stratification in the myelin sheath was more obvious where there were black particles accumulated (Fig. 3B). The myelinated fibres were dissociated, and the inherent structure was destroyed, by which to say it had a swollen state as compared to the structure of local myelin. Moreover, black particles were only aggregated in the myelin.

Some pathogenic changes in the brain at $104 \mathrm{dpc}$ are shown in Fig. 4. Deposition or polymerisation of the particles was much more evident, yet they were still limited within the myelin sheath. Some part of the myelin had dissolved and the damage was more significant, including lysis of the plasma membrane (Fig. 4A, rightward arrow). Bubbly structures (Fig. 4A, leftward arrow) had appeared, yet organelle damage was not obvious. Degeneration was more likely to have happened at the border between two myelin sheaths (Fig. 4B, rightward arrow), and adjacent mitochondria were also damaged due to deterioration of the myelin (Fig. 4B, downward arrow).

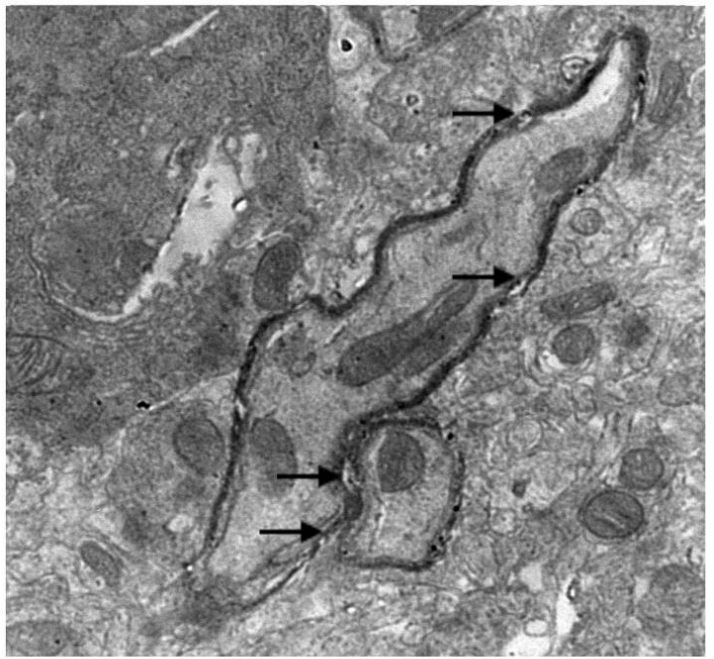

A. $3000 x$

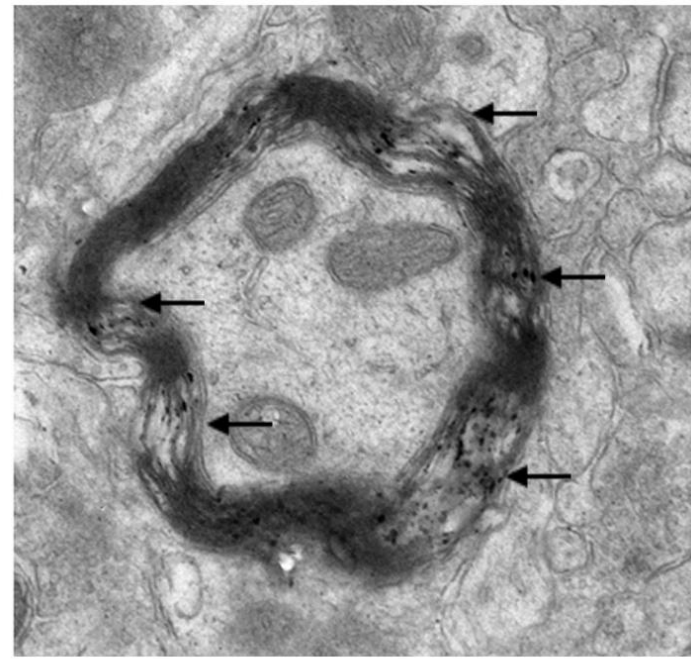

B. $8000 \times$

Fig. 3. The myelinated nerves of mouse brain from the test group at 97 days post challenge. The myelin had become curved and deposition or polymerisation of dispersed particles was obvious in the myelin sheath. Rightward arrows indicate that the cytoplasmic membrane was no longer integrated and leftward arrows indicate that stratification in the myelin sheath was more obvious where there were black pathogen-like particles accumulated

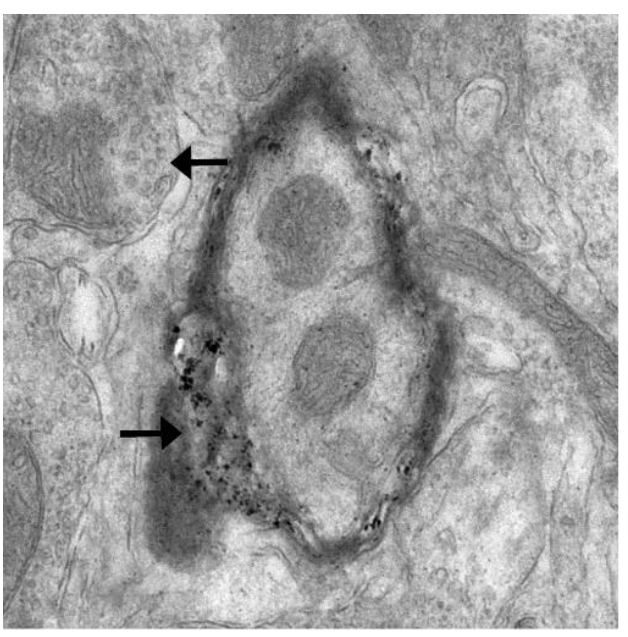

A. $8000 \times$

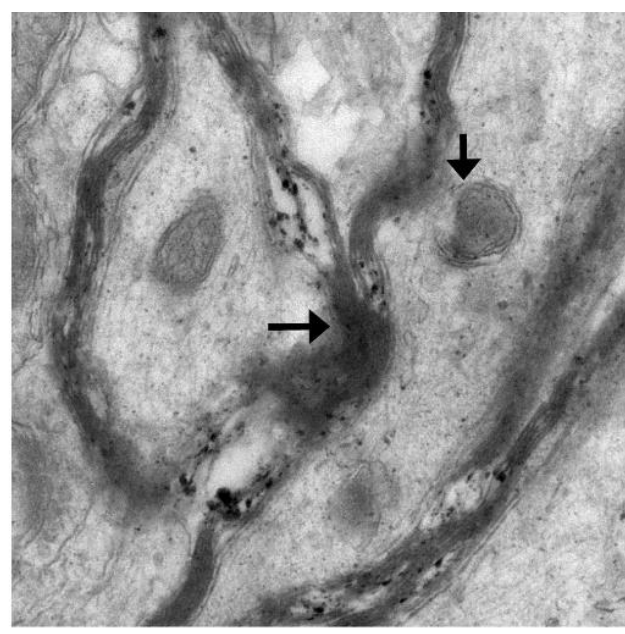

B. $10000 \times$

Fig. 4. The myelinated nerves of mouse brain from the test group at 104 days post challenge. The plasma membrane had dissolved and spread out like mud (rightward arrow). In addition, there were bubbly structures (leftward arrow). Degenerative changes at the border between two myelin sheaths were more obvious (rightward arrow) and adjacent mitochondria are also damaged (downward arrow) 
At $111 \mathrm{dpc}$, the cytoplasmic membrane was separated or destroyed, myelin detached from the main body, and there were large black particles accumulated. Furthermore, the polymeric particles were also present in the polymerised bubbles along with the detached myelin sheath (Fig. 5A, downward arrow). The myelin was disintegrated and scattered in the medullary cavity where some black particles were also aggregated. The structure of mitochondria was almost normal, bubbly structures had appeared once again (Fig. 5B), and there were suspicious pathogenic fibres (Fig. 5A, upward arrow).

At $118 \mathrm{dpc}$, pathological changes had occurred mainly in the myelin sheath (Fig. 6). It had become curved and a multilayer fiber layer had formed. Stratification was one of the significant characteristics in the local myelin sheath along with the aggregation of black particles. These deposited particles were bound with the solitary fibrous layer, and no free small and uniform black particles were observed. The structure of the myelin sheath was still integrated, with a slightly fuzzy appearance, and mitochondria were roughly intact. However, the myelin sheath had separated from the plasma membrane and a large gap had formed between two myelin sheaths. The aggregated black particles were mainly located in the lamination of the myelin sheath, and whether these black particles could become obvious seemed to be associated with the stratification extent. From $118 \mathrm{dpc}$, this stratification effect gradually diffused to both sides of the myelin sheath. The cytoplasm was like silk-slurry, while some specimens showed multiple sets of annular structure. Conformation changes in the myelin did not appear simultaneously, but usually these changes were more obvious in the adjacent dendritic myelin. There were widespread abnormal pathogenic structures at $118 \mathrm{dpc}$ such as local extension, bending, and structural damage in the myelin sheath (Fig. 6). There were also many membrane cavities (autophagy vesicles) containing a membrane vacuole (Fig. 6A). Small black or specific particles were scattered among cells and tubulovesicular structures could also be seen (Fig. 6B).

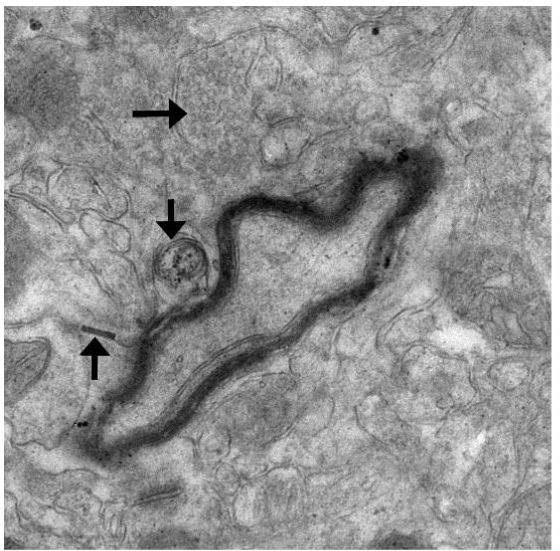

A. $8000 \times$

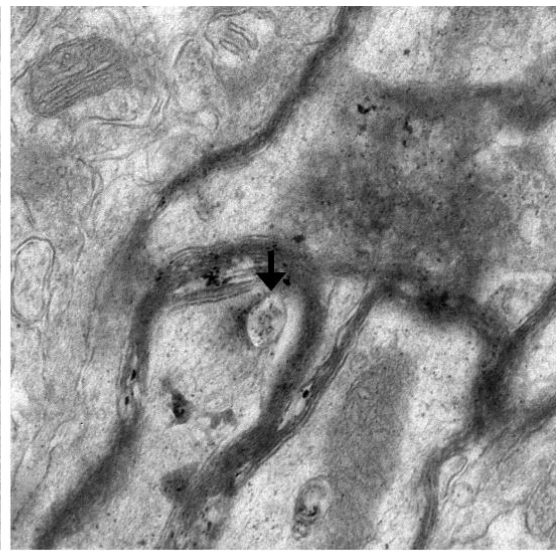

B. $7000 \times$

Fig. 5. The myelinated nerves of mouse brain from the test group at 111 days post challenge. The rightward arrow points to the bubbly structures, the upward arrow points suspicious pathogenic fibres, and the downward arrows point to the polymerised bubble with polymeric particles

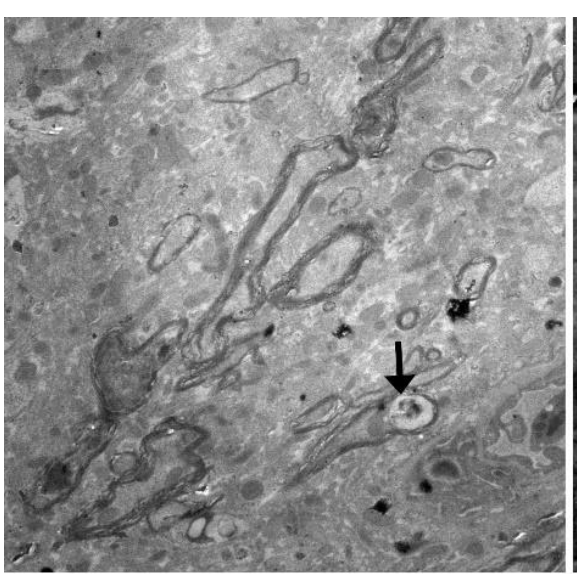

A. $1200 \times$

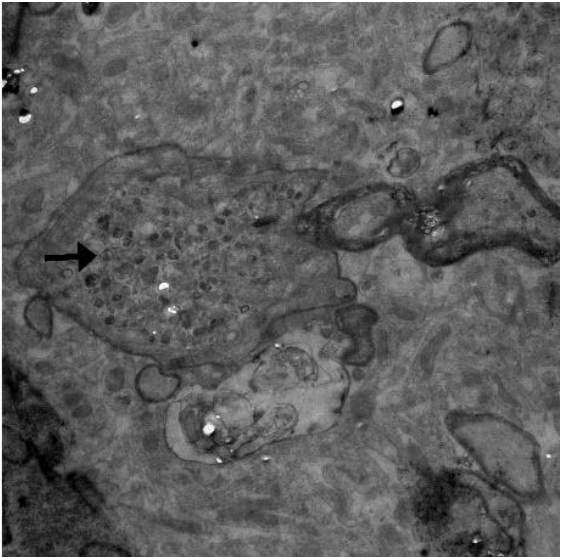

B. $1500 \times$

Fig. 6. The myelinated nerves of mouse brain from the test group at 118 days post challenge. Some abnormal pathogenic structures like local extension, bending, and black particle deposition were widespread in the myelin sheath. The downward arrow indicates membrane vacuole, while the rightward arrow indicates tubulovesicular structure 
At $125 \mathrm{dpc}$, stratification in the myelin sheath had increased and become a universal abnormal structure. In addition, some myelin sheaths had become swollen and diffused (Fig. 7) and more particles had been deposited in local myelin. However, pathological changes were still limited to the myelin sheath and had rarely spread to the cytoplasm.

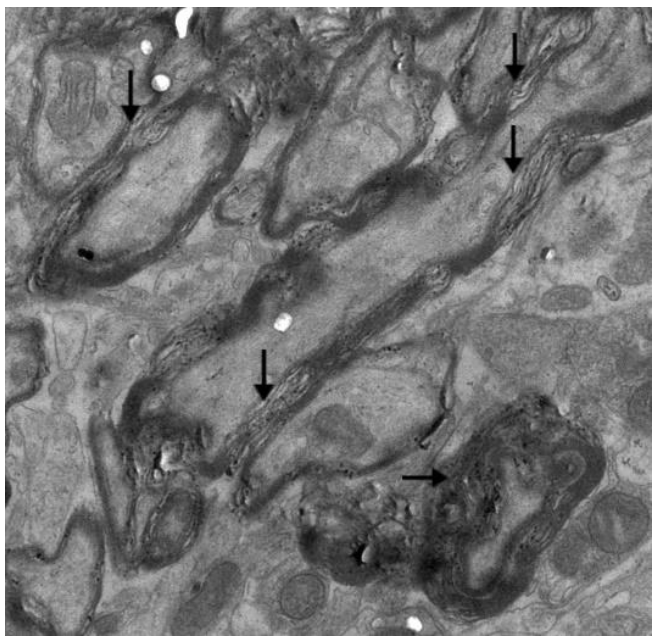

Fig. 7. The myelinated nerves of mouse brain from the test group at 125 days post challenge. The stratification in the myelin sheath had increased as indicated by downward arrows, and some myelin sheaths had swollen and become diffused as indicated by the rightward arrow. $1200 \times$

At $132 \mathrm{dpc}$, particle aggregation and stratified myelin were commonly observed features. The aggregated particles were distributed uniformly in the myelin sheath. The collapsed myelin sheath had spread to the cytoplasm and the infolded myelin sheath had further developed into phagocytic vacuoles (Fig. 8A). The typical Wallerian degeneration and intramyelinic vacuoles were obvious in the myelin sheath, while some myelin sheaths had formed multi-membrane vacuoles (Fig. 8B), in which the aggregated black particles were gradually dissolving. In the normal myelin, the aggregated black particles were not found, and the structures of cytoplasm and organelles were barely changed.

At $139 \mathrm{dpc}$, most of the dendrite myelin sheaths had swollen and become stratified, and particle aggregation or deposition was much more obvious than before (Fig. 9). The aggregated dark particles were gradually dissolving and further developing into a multivacuole structure, in which nothing tangible could be seen. Vacuoles or large areas of dissolved plaques had appeared outside the myelin. Polymeric particles attached to the myelin fibres were still visible. It was found that one myelin sheath contained another (Fig. 9A), and mitochondria had swollen conspicuously. In addition, both particle polymerisation and myelin sheath lamination had sharply increased (Fig. 9B). Although myelin still maintained its original shape, these changes had affected the basic form of cytoplasm, which had partly or completely dissolved. The black particles in the myelin sheath had diffused into the cytoplasm in relatively free forms. External tissues of the myelin sheath also showed the dissolved plaques.

At $146 \mathrm{dpc}$, the dark particle aggregations were more obvious in the myelin sheath, and some polymerised particles were in free forms, while most of them were attached to the myelin fibres. The cytoplasm was fully dissolved and multilayer membrane vesicles (large autophagy vacuoles) could also be seen. The whole myelin sheath cavity was filled with vacuoles and there was abundant proliferation of layered myelin fibres. The polymeric particles had become larger and there was myelin in a sleeve type or even in a four-layer casing pipe (Fig. 10, downward arrow). Black polymeric particles had increased obviously and were deposited in the myelin. The intracellular mitochondrial structure was integrated, but the myelin sheath was separated from the membrane (Fig. 10, upward arrows). It can be conclusively asseverated that the pathological changes in the brain infected with $\operatorname{PrP}^{\mathrm{Sc}}$ first occur in the myelin sheath, and then in cytoplasm or external tissues.

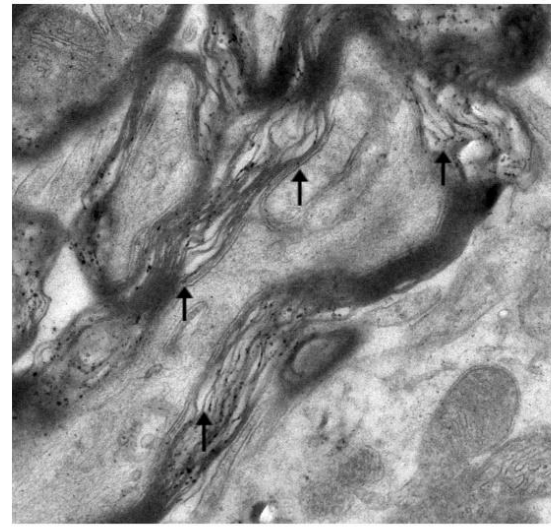

A. $6000 \times$

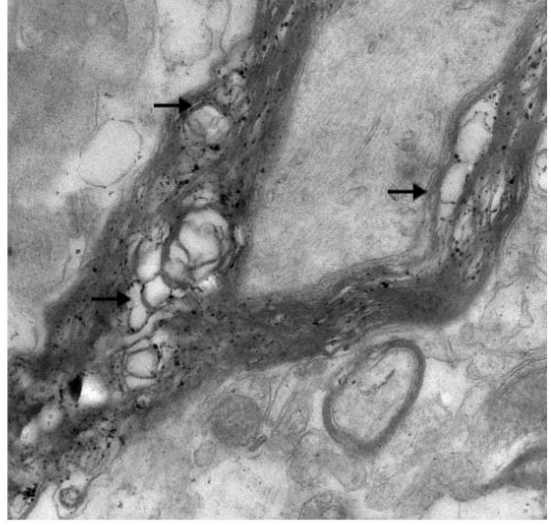

B. $5000 \times$

Fig. 8. The myelinated nerves of mouse brain from the test group at 132 days post challenge. Upward arrows indicate that the myelin sheath had collapsed and spread to the cytoplasm and the infolded myelin sheath had further developed into phagocytic vacuoles and rightward arrows indicate that multi-membrane vacuoles had formed in some myelin sheaths and the aggregated black particles were dissolving in these vacuoles 


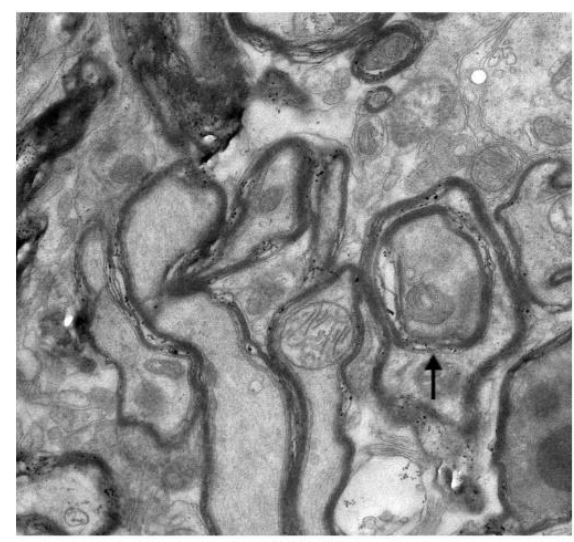

A. $3000 \times$

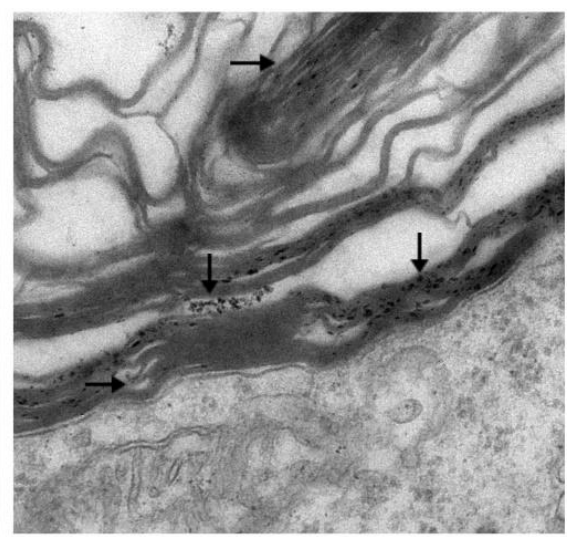

B. $12000 \times$

Fig. 9. The myelinated nerves of mouse brain from the test group at 139 days post challenge. The myelin sheath is stratified obviously and many more particle aggregations or depositions were observed. The upward arrow indicates that one myelin sheath contained another downward arrows indicate particle polymerisation, and rightward arrows indicate myelin sheath lamination
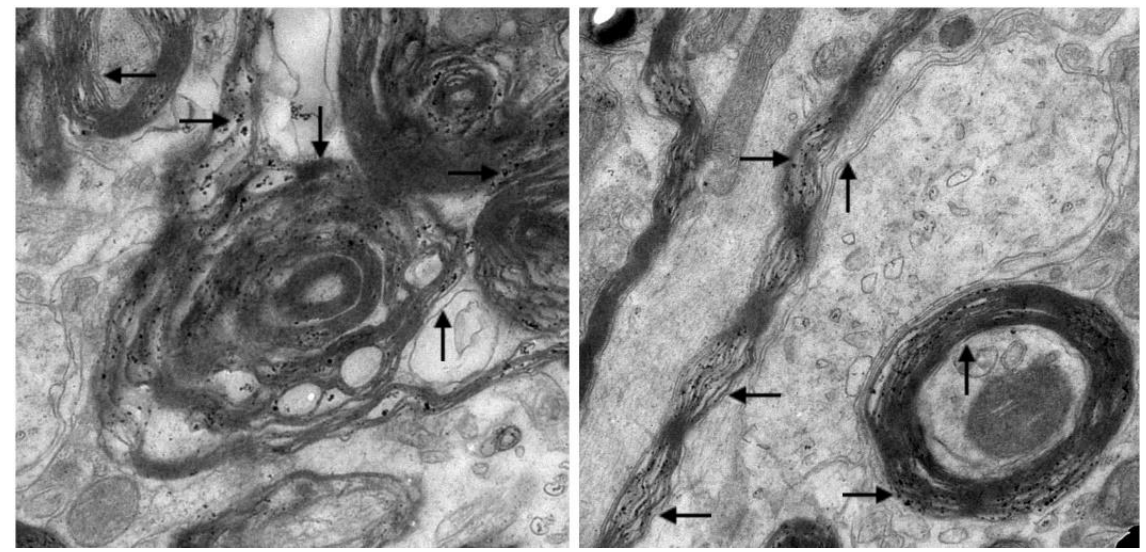

Fig. 10. The myelinated nerves of mouse brains from the test group at 146 days post challenge. The whole myelin sheath cavity is filled with vacuoles and layered myelin fibres, and there is typical particle deposition, myelin stratification, and vacuolisation. The downward arrow indicates the myelin in the form of a four-layer pipe, upward arrows indicate the myelin sheath separated from the membrane, rightward arrows indicate particle polymerisation and deposition, and leftward arrows indicate myelin sheath lamination. $4000 \times$

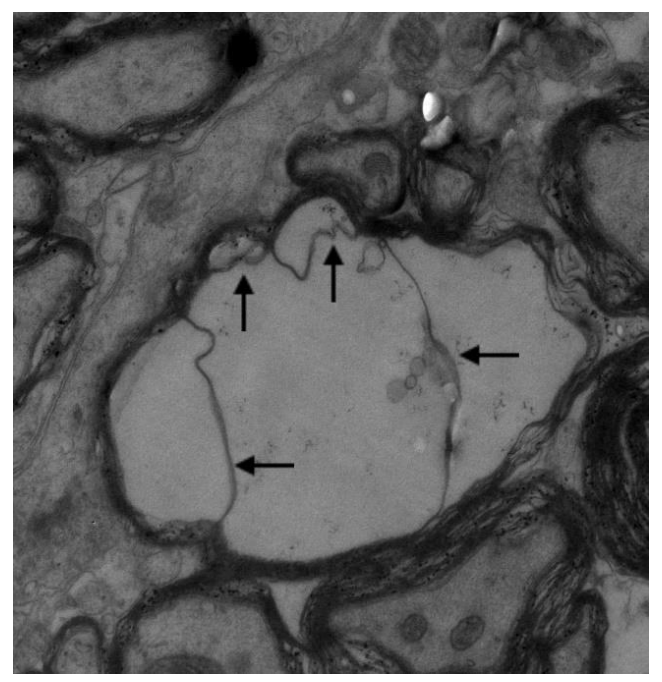

Fig. 11. The myelinated nerves of mouse brain from the test group at 153 days post challenge. The cytoplasm had completely dissolved and separated from the membrane as indicated by leftward arrows. Upward arrows indicate that the detached axonal membrane had formed an inner tongue. $3000 \times$
At $153 \mathrm{dpc}$, the myelin sheath was further stratified and black particles much more aggregated. The cytoplasm had completely dissolved and separated from the membrane, and thus gave the appearance of vacuolisation. The axonal membrane had broken away from the myelin or proliferated to form an inner tongue (Fig. 11).

At $160 \mathrm{dpc}$, myelin stratification was still in progress and myelin had become curved due to the lack of tension (Fig. 12). The myelin detachment had caused vacuolisation of cytoplasm while the disaggregation of local myelin had led to the production of multiple vesicles. Almost all the myelin sheath was stratified and had swollen and become multi-hierarchical. Moreover, lesions were diffused outward, and part of the cytoplasm and extra-cellular structures had dissolved to form plaques. The mitochondria had swollen and become fuzzy, and adjacent structures of the cytoplasm and myelin were almost illegible. There were analogous autophagocytic vacuoles and multimembrane vesicles had appeared in the myelin 


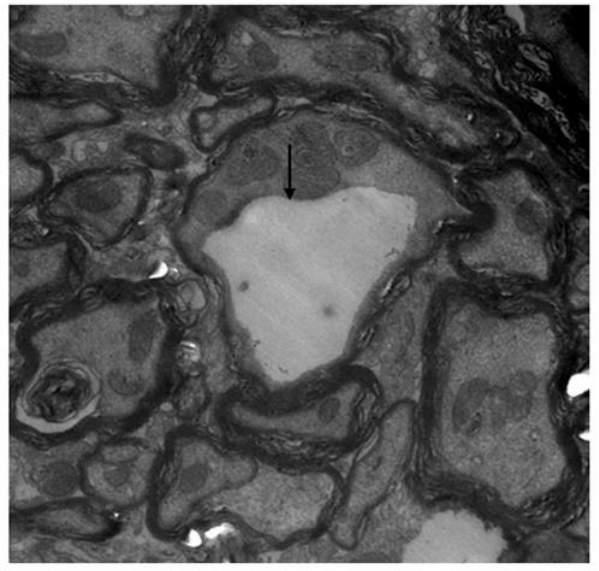

A. $2000 \times$

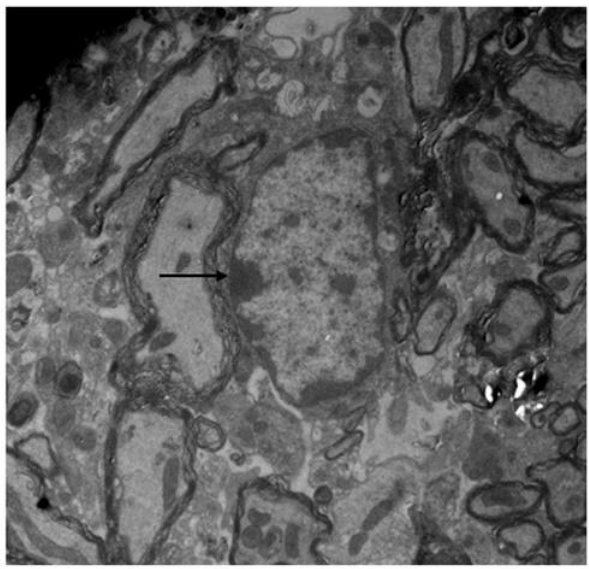

B. $1200 \times$

Fig. 12. The myelinated nerves of mouse brain from the test group at 160 days post challenge. There were autophagocytic vacuoles and multimembrane vesicles in the myelin sheath, polymeric particles had become still more blurred, and the aggregated black particles in the myelin had almost dissolved. The downward arrow indicates the large dissolved plaques and the rightward arrow indicates that macrophagocyte nuclei were marginally assembled inside cells

sheath, large dissolved plaques were present (Fig. 12A), and the myelin close to plaques had disintegrated severely. With the continuous development of the pathological changes, polymeric particles had become more and more blurry, while the aggregated black particles in the myelin had almost dissolved to form plaque. Damage to the myelin had gradually expanded resulting in the disintegration and dissolution of myelin and cytoplasm respectively. However, most of the mitochondria still retained an intact structure. Membrane invagination and dissolved plaques exterior to the cytoplasm could also be seen. Nuclei of macrophagocytes were marginally assembled inside the cells (Fig. 12B). When the mice were on the verge of death, the nerve fibres had changed abnormally and inconsistently. However, stratification in the myelin sheath and aggregation of black particles were still existent. These black particles were considered universal pathological changes.

\section{Discussion}

Many potential roles and ligands for $\mathrm{PrP}^{\mathrm{C}}$ have been reported. Those studies suggest that $\operatorname{PrP}^{\mathrm{C}}$ may act as a scaffolding protein taking part in some cell surface interactions and signalling mechanisms. $\operatorname{PrP}^{\mathrm{C}}$, an anchor protein (GPI) or a surface glycoprotein, is located on the extracellular surface and circulates between the plasma membrane and endocytic compartment. In addition, it participates in many biological processes of functional proteins, such as that of clathrin-plasma membrane associated caveolaevesicle during the circulation process of cell membrane. Meanwhile, $\operatorname{PrP}^{\mathrm{C}}$ is an essential structure for the endocytosis of higher central nerve fibre content on the surface than other cells. The GPI of $\operatorname{PrP}^{\mathrm{C}}$ directly reacts with clathrin leading to cell invagination into the protoplasma. Several studies have implied that the conversion of $\mathrm{PrP}^{\mathrm{C}}$ to $\mathrm{PrP}^{\mathrm{Sc}}$ may take place during this process of invagination, and glycosylation of $\mathrm{PrP}^{\mathrm{C}}$ is the key to this misfolded transformation (29). In this transformation process, the exogenous $\mathrm{PrP}^{\mathrm{Sc}}$ is required as an incentive, and normal $\operatorname{PrP}^{\mathrm{C}}$ participates as the substrate to be transformed into an isoform or as a pathogenic factor (25). When this transforming process occurs in neuronal cells, it occurs mainly in the myelin sheath. Hence, the myelin sheath is the main site for prion conversion, which has been verified in the present study.

The normal structure of the myelin sheath of nerve fibres is compact with all laminations closely combined or the lipid rafts showing a laminated structure. The whole myelin is closely tied to the cytoplasmic membrane and the entire nerve fibre is orderly and tightly arranged. However, per observations of the brain pathological lesions of BALB/c mice infected with prion RML strain for 160 days, black particles first appeared in the myelin sheath of axons and dendrites or in lipid rafts. These black particles spread inside or outside the cell, and organelles such as the mitochondria were involved in further lesions that occurred in the myelin sheath. These findings indicated that the myelin sheath and lipid rafts were the first or main sites where the prion protein changed conformation (28). $\operatorname{PrP}^{\mathrm{C}}$ was expressed at a higher level in nerve cells especially in the myelin sheath, which may result from induction and serious pathological lesions caused by $\operatorname{PrP}^{\mathrm{Sc}}$ invasion. Besides this, $\operatorname{PrP}^{\mathrm{C}}$ was the main source of the substrate for abnormal transformation of $\operatorname{PrP}^{\mathrm{C}}$ induced by $\mathrm{PrP}^{\mathrm{Sc}}$. Therefore, the myelin sheath with its high content of $\operatorname{PrP}^{\mathrm{C}}$ may become the main place where pathogenic $\mathrm{PrP}^{\mathrm{Sc}}$ is formed. In this study, we observed the lesions in mouse brain that were directly injected with prion proteins 
contained in brain homogenates. Results revealed that characteristic black particles were polymerised in the myelin sheath, which was not reported in previous studies. During the long period, all pathological lesions of the brain induced by $\operatorname{PrP}^{\mathrm{Sc}}$ were continuously and systematically observed $(1,3)$. Different pathogenic strains would lead to different responses of the host (18). In the present study, prion strain RML was found to induce black particle aggregations in the myelin sheath, and in parallel with the advance of the infection, the deposition and aggregation of the black particles were much more obvious at successive intervals.

Some collapsed axons were surrounded by abnormal condensed multilamellar myelin sheaths in some nerve fibres, which strongly corresponded to an earlier study (3). Myelin sheaths with uniform or broken stratification were observed in the brain of mice injected with normal mouse brain homogenates and saline respectively, yet there were no black particles. The major structure of the myelin sheath is a lamination, while prion protein also has a lamellar conformation. $\mathrm{PrP}^{\mathrm{C}}$ is a membrane-bound glycoprotein, which mainly consists of an $\alpha$-sheet layer and a $\beta$-sheet layer. However, the $\beta$-sheet of the lamellar structure is disordered as prion protein polymerisation occurs under pathological conditions (27).

It was supposed that the myelin sheath and plasma membrane were the key locations of $\mathrm{PrP}^{\mathrm{Sc}}$ transformation as reported in earlier studies (18). According to the $\operatorname{PrP}$ endocytosis theory, $\operatorname{PrP}^{\mathrm{Sc}}$ molecules first anchor on the surface of the membrane outside the myelin sheath, and then they may bend or invaginate when they encounter and bind with the myelin. For example, the myelin sheath becomes curved and its tensile strength is insufficient to keep it rigid and straight, as seen in the present and other studies (11). The sites undergoing bending or invagination were often the starting points of particle aggregations that subsequently led the myelin sheath to delaminate, as confirmed in many previous studies (26). Particle aggregations were the primary pathological lesions presented within the myelin sheath regardless of the structural changes in myelin. The black particles do not spread to the cytoplasm or outside the cell, possibly because the overflowing polymeric particles are dispersed or dissolved when they are in a different environment. Perhaps the potential locations for the conversion of $\operatorname{PrP}^{\mathrm{C}}$ to $\operatorname{PrP}^{\mathrm{Sc}}$ are related to the myelin membrane, amyloid plaques, pathways from endocytosis to lysosomes, interactive contact points between neurites, dendritic cells in the spleen and tonsil, etc. (25). The binding of $\mathrm{PrP}^{\mathrm{Sc}}$ with the membrane receptor may change the local environment of the myelin, which may then cause the failing of $\mathrm{PrP}^{\mathrm{C}}$ glycosylation right after $\mathrm{PrP}^{\mathrm{Sc}}$ anchors $\mathrm{PrP}^{\mathrm{C}}$ on the membrane. Secondly, non-glycosylated $\operatorname{PrP}^{\mathrm{C}}$ may be more easily polymerised and transformed into pathogenic $\operatorname{PrP}^{\mathrm{Sc}}$ using $\mathrm{PrP}^{\mathrm{Sc}}$ as the template.
Since configuration shift or transformation of protein may depend on the environmental $\mathrm{pH}$, the configuration of $\mathrm{PrP}^{\mathrm{Sc}}$ formed at $\mathrm{pH}$ 4-6 shows stronger resistance to protease than its counterpart formed at $\mathrm{pH}$ 7.4. Low $\mathrm{pH}$ may stimulate the conversion of the secondary structure from $\operatorname{PrP}^{\mathrm{C}}$ to $\mathrm{PrP}^{\mathrm{Sc}}$, and prion protein should be insoluble at $\mathrm{pH}$ below 3.5. As a result, configuration changes of $\mathrm{PrP}^{\mathrm{Sc}}$ must be $\mathrm{pH}$ dependent, and tend towards the $\beta$-sheet by blocking the digestion sites of the protease at $\mathrm{pH}$ below 4.0. The configuration conversion of $\mathrm{PrP}^{\mathrm{Sc}}$ is mainly caused by $\mathrm{N}$-terminal infolding or invagination (9).

It is hardly possible for $\mathrm{PrP}^{\mathrm{Sc}}$ to be internalised after combining with other chaperone molecules as the resulting structures are too large. Perhaps $\mathrm{PrP}^{\mathrm{Sc}}$ could react with different types of trans-membrane ligands on various cell membranes and the resultant reaction between $\operatorname{PrP}^{\mathrm{C}}$ and $\mathrm{PrP}^{\mathrm{Sc}}$ may convert the normal $\mathrm{PrP}^{\mathrm{C}}$ into $\operatorname{PrP}^{\mathrm{Sc}}(21)$. When $\operatorname{PrP}^{\mathrm{Sc}}$ appears on the cell myelin sheath, it usually shows decreased membrane tension, buckling, invagination, caveolae, and increased folding and stratification. These could be related to the change in the membrane structure, which is caused by the polymerisation of $\mathrm{PrP}^{\mathrm{C}}$ and $\mathrm{PrP}^{\mathrm{Sc}}$ complexes. Exogenous $\mathrm{PrP}^{\mathrm{Sc}}$ must be internalised into the cell membrane in order to infect new cells. In this case, $\mathrm{PrP}^{\mathrm{Sc}}$ is able to contact $\operatorname{PrP}^{\mathrm{C}}$ mainly on the molecular surface at the C-terminal, however, this process is limited by the specificity of prion protein GPI binding to the membrane. Therefore, $\operatorname{PrP}^{\mathrm{C}}$ may be considered a receptor for the internalisation of $\mathrm{PrP}^{\mathrm{Sc}}$ into the cell. In other words, $\mathrm{PrP}^{\mathrm{Sc}}$ is not able to infect all kinds of animals (24) as the specificity of $\mathrm{PrP}^{\mathrm{C}}$ involves the species barrier.

Several studies reported that deglycosylated or incompletely glycosylated $\mathrm{PrP}^{\mathrm{C}}$ is much easier to convert into $\mathrm{PrP}^{\mathrm{Sc}}$ (32). The glycosylation process would be hampered after $\operatorname{PrP}^{\mathrm{C}}$ binding with $\mathrm{PrP}^{\mathrm{Sc}}$, and non-glycosylated molecules are prone to accumulate and change configuration, which may also cause mutation in the sequence of $\mathrm{PrP}^{\mathrm{C}}$ and then transformation to $\operatorname{PrP}^{\mathrm{Sc}}$ (10). Several scientists artificially performed deglycosylation of $\operatorname{PrP}^{\mathrm{C}}$ and as a result, $\operatorname{PrP}^{\mathrm{C}}$ accumulated in the cytosol and converted into pathogenic $\mathrm{PrP}^{\mathrm{Sc}}$. Hence, glycosylation may regulate the configuration changes between $\operatorname{PrP}^{\mathrm{C}}$ and $\mathrm{PrP}^{\mathrm{Sc}}$ (32). Aggregated particles could also be seen in other experiments as well, but they were not so typical as in our test and so failed to draw the scientists' attention (19). The characteristic findings of the particle aggregations in the myelin were more obvious and strongly consistent with several other results $(20,26)$. However, the multilayer structure and herniation of the myelin formed cannot be explained in the present study. It may be caused by a change in membrane structure or be due to the alteration of polymeric patterns (12).

At day 90, the mice started to show clinical symptoms such as itching and depression. Therefore, 
the changes in their brains were observed beginning from $90 \mathrm{dpc}$, which was a key time point judging from a series of pathological lesions. In the initial stage, the configuration-changed $\operatorname{PrP}^{\mathrm{C}}$ in nerve fibres was polymerised as seeds and the misfolded proteins caused configuration disorder. From day 90, similar changes occurred; abnormal $\mathrm{PrP}^{\mathrm{C}}$ proteins were polymerised directly and led to the continuous appearance of misfolded proteins. As the proteasomes controlling this kind of misfolded proteins were over-burdened, this led to the formation of abnormal $\mathrm{PrP}^{\mathrm{C}}$ protein polymers, the deglycosylated proteins occupying the dominant places, and myelin layering or hyperplasia $(25,28)$. However, the aggregation of suspicious black particles did not appear, they presented with dissolved or disintegrated forms; we supposed it may be caused by autophagy, proteasome, and inner environmental alterations. These kinds of processes were not really observed very clearly in our experiments, except autophagy that was formed through axoplasm dissected by the cytoplasmic membrane. The polymer of $\mathrm{PrP}^{\mathrm{C}}$ with the resistant structures of a protease repressor furnished genuine proof that the normal prion proteins could be changed into abnormal prion proteins. Meanwhile, mixing $\mathrm{PrP}^{\mathrm{Sc}}$ and $\operatorname{PrP}^{\mathrm{C}}$ in vitro would start to convert $\operatorname{PrP}^{\mathrm{C}}$ to $\operatorname{PrP}^{\mathrm{Sc}}$ under certain conditions (2). The polymerised $\operatorname{PrP}^{\mathrm{C}}$ was as toxic to cells as some varieties of similar misfolded proteins, such as membrane-protein-1, presenilin-1, cystic-fibrosis transmembrane conductance regulator (CFTR), and parkin protein. The prion protein was not dissolved but became polymer-like particles in the myelin, while it was soluble in cytoplasm. The damage to the peripheral organelles caused by aggregation of suspicious black particles occurred gradually in the myelin sheath. It may have been the combination of the proliferation of $\mathrm{PrP}^{\mathrm{Sc}}$ with myelin bending, changes of tension, and inner environmental factors which jointly delaminated and aggregated black particles in the myelin sheath. When the layered structure of myelin was completely destroyed along with the gradual homogenisation or dissolution of the suspicious black particle aggregations, pathological lesions would gradually expand to the periphery.

The changes and degeneration between the two myelin sheaths were more violent or faster, which may be due to the fact that more prion proteins were attracted by the prion anchor and more misfolded proteins interacted with each other. In this study, the classical lesions in the nerves were observed, including Wallerian degeneration, myelinate fibre formation, and intramyelinic vacuoles. With the increase of $\mathrm{PrP}^{\mathrm{Sc}}$ in the myelin, the structures of the myelin sheath and plasma membrane were destroyed and then $\mathrm{PrP}^{\mathrm{Sc}}$ diffused into the cells and the surroundings, finally causing the degradation of proteasome and autophagic vacuoles in axoplasm. The organelles may be digested by autophagic vacuoles, leading to death of cells. The degenerated nerve fibres in the experiment had actually lost their normal function, and in clinical examination the mice showed symptoms such as itching (7).

The most obvious pathological lesions observed in mouse brain were the aggregation of particles and stratification in the myelin sheath after infection for 90 days. The emergence of polymeric particles may be caused by $\operatorname{PrP}^{\mathrm{Sc}}$ induction, comprehensive effects of environment within the myelin, and a variety of auxiliary proteins or other molecules. It has been speculated that the conversion of $\operatorname{PrP}^{\mathrm{C}}$ to $\operatorname{PrP}^{\mathrm{Sc}}$ was just a configuration change of proteins without the involvement of nucleic acids $(14,30)$.

As for the pathogenicity of the polymeric protein, orderly aggregated pathogens were highly infectious. The $\mathrm{PrP}^{\mathrm{Sc}}$ in monomeric form was usually harmless, and yet it might be prone to convert into the primary $\mathrm{PrP}^{\mathrm{Sc}}$ aggregates. $\mathrm{PrP}^{\mathrm{Sc}}$ polymers consisted of less than 20 molecules with higher toxicity and faster diffusion ability (2). Thus, prion strain RML should be a highly toxic strain, as no big polymers were observed in the myelin sheath. Toxicity of the low polymerised $\mathrm{PrP}^{\mathrm{Sc}}$ was first manifested in the dysfunctional nerve fibre membrane, extremely swollen myelin, and induction of disintegration and apoptosis of cells. $\mathrm{PrP}^{\mathrm{Sc}}$ is the pathogenic factor of several prions, and its pathogenesis may be different from other prions. The diffusion of polymeric particles has still not been elucidated but may be due to various environmental $\mathrm{pH}$ and enzyme activities. The internal environment of the myelin sheath is conducive to the formation of $\mathrm{PrP}^{\mathrm{Sc}}$ and protein misfolding.

$\mathrm{PrP}^{\mathrm{Sc}}$ would eventually reach the brain through either gastrointestinal ingestion or injection into the body, and cause deadly damage to the central nervous system. It was thought that $\operatorname{PrP}^{\mathrm{Sc}}$ was transmitted by the axoplasm of nerve fibres and synapses $(2,28)$. We speculated that the possible transmission manner of $\mathrm{PrP}^{\mathrm{Sc}}$ was along the myelin sheath by other means than axoplasm. The transmission speed of $\mathrm{PrP}^{\mathrm{Sc}}$ in the axonal cytoplasm membrane cavity is $0.5-3 \mathrm{~mm} /$ day (28). Alternatively, the transmission between cells is through endocytosis, and the denatured prion proteins are aggregated into a stable complex. It is gradually transferred along the nerve sheath, possibly through the domino effect (28).

Prion aggregation may be caused by environmental changes, for the cells change their structures in response to specific variations of environmental factors. In this study, the aggregate particles are observed, and we think $\mathrm{PrP}^{\mathrm{sc}}$ is the causative factor, which has also been found in bacteria of prokaryotic cells (35). So, the structure change of prions may be a universal phenomenon in biology. The previous research proved that the two key positions (23 and $12 \mathrm{bp}$ indels) are sensitive sites where potentially the transformation of $\operatorname{PrP}$ into $\operatorname{PrP}^{\text {sc }}$ could be stimulated $(8,17,22,33)$. In our experiment, we found the myelin started to polymerise from one site and then gradually 
spread by domino effect, which may be associated with the excitation sites. Yuan et al. (35) found the structure transformation of prion after inserting a section of prion into the bacterial membrane, while we observed that the transformation might start from myelin sheath in mice. The inability to see the polymeric particles in the cytoplasm may be attributed to the dissolved state.

In summary, electron microscope observation showed widespread presence of particle aggregations and delamination in the axons and dendrites of the myelin sheath in the brain of BALB/c mice injected with pathogenic brain homogenates. Aggregation of particles and stratification occurred much more easily in the adjacent myelin, which demonstrated that the conformational change of prion protein was more likely to occur in the myelin sheath and not in cytoplasm or outside the cells.

Conflict of Interests Statement: The authors declare that there is no conflict of interests regarding the publication of this article.

Financial Disclosure Statement: This work was supported by the National Natural Science Foundation of China (Grant. 30871956); and the Science \& Technology Development Project of Jilin Province, China (Grant 0170101149JC and 20170101035JC).

Animal Rights Statement: The authors declare that all experiments on animals were conducted in accordance with the provisions of EU animal management practices under controllable situations and the Jilin university ethical committee guidelines for the Care and Use of Laboratory Animals.

Acknowledgements: Both Dr. Shu-Sen Cui (sscui916@126.com) and Dr. Hong-Lin Ren (renhl@jlu.edu.cn) are the corresponding authors. We appreciate the work of all authors in designing, performing and composing this study.

\section{References}

1. Agostini F., Dotti C.G., Perez-Canamas A., Ledesma M.D., Benetti F., Legname G.: Prion protein accumulation in lipid rafts of mouse aging brain. PLoS One 2013, 8, e74244.

2. Aguzzi A., Calella A.M.: Prions: protein aggregation and infectious diseases. Physiol Rev 2009, 89, 1105-1152.

3. Baumann F., Tolnay M., Brabeck C., Pahnke J., Kloz U., Niemann H.H., Heikenwalder M., Rulicke T., Burkle A., Aguzzi A.: Lethal recessive myelin toxicity of prion protein lacking its central domain. EMBO J 2007, 26, 538-547.

4. Brandner S., Raeber A., Sailer A., Blattler T., Fischer M., Weissmann C., Aguzzi A.: Normal host prion protein (PrPC) is required for scrapie spread within the central nervous system. Proc Natl Acad Sci USA 1996, 93, 13148-13151.

5. Bueler H., Fischer M., Lang Y., Bluethmann H., Lipp H.P., DeArmond S.J., Prusiner, S.B., Aguet M., Weissmann C.: Normal development and behaviour of mice lacking the neuronal cell-surface PrP protein. Nature 1992, 356, 577-582.
6. Chen W., van der Kamp M.W., Daggett V.: Diverse effects on the native beta-sheet of the human prion protein due to diseaseassociated mutations. Biochemistry 2010, 49, 9874-9881.

7. Conforti L., Gilley J., Coleman M.P.: Wallerian degeneration: an emerging axon death pathway linking injury and disease. Nat Rev Neurosci 2014, 15, 394-409.

8. Czarnik U., Strychalski J., Zabolewicz T., Pareek C.S.: Populationwide investigation of two indel polymorphisms at the prion protein gene in Polish Holstein-Friesian cattle. Biochem Genet 2011, 49, 303-312.

9. DeMarco M.L., Daggett V.: Local environmental effects on the structure of the prion protein. C R Biol 2005, 328, 847-862.

10. Dickinson A.G., Outram G.W.: Genetic aspects of unconventional virus infections: the basis of the virino hypothesis. Ciba Found Symp 1988, 135, 63-83.

11. Ersdal C., Goodsir C.M., Simmons M.M., McGovern G., Jeffrey M.: Abnormal prion protein is associated with changes of plasma membranes and endocytosis in bovine spongiform encephalopathy (BSE)-affected cattle brains. Neuropathol Appl Neurobiol 2009, 35, 259-271.

12. Ersdal C., Simmons M.M., Goodsir C., Martin S., Jeffrey, M.: Sub-cellular pathology of scrapie: coated pits are increased in PrP codon 136 alanine homozygous scrapie-affected sheep. Acta Neuropathol 2003, 106, 17-28.

13. Flannigan D.J., Zewail A.H.: 4D electron microscopy: principles and applications. Acc Chem Res 2012, 45, 1828-1839.

14. Frid K., Einstein O., Friedman-Levi Y., Binyamin O., BenHur T., Gabizon R.: Aggregation of MBP in chronic demyelination. Ann Clin Transl Neurol 2015, 2, 711-721.

15. Fujita M., Kinoshita T.: Structural remodeling of GPI anchors during biosynthesis and after attachment to proteins. FEBS Lett 2010, 584, 1670-1677.

16. Gul N., Ozkorkmaz E.G., Kelesoglu I., Ozluk A.: An ultrastructural study, effects of Proteus vulgaris OX19 on the rabbit spleen cells. Micron 2013, 44, 133-136.

17. Gurgul A., Czarnik U., Larska M., Polak M.P., Strychalski J., Slota E.: Polymorphism of the prion protein gene (PRNP) in Polish cattle affected by classical bovine spongiform encephalopathy. Mol Biol Rep 2012, 39, 5211-5217.

18. Horonchik L., Tzaban S., Ben-Zaken O., Yedidia Y., Rouvinski A., Papy-Garcia D., Barritault D., Vlodavsky I., Taraboulos A.: Heparan sulfate is a cellular receptor for purified infectious prions. J Biol Chem 2005, 280, 17062-17067.

19. Jeffrey M., Goodsir C.M., Bruce M., McBride P.A., Scott J.R., Halliday W.G.: Correlative light and electron microscopy studies of $\operatorname{PrP}$ localisation in $87 \mathrm{~V}$ scrapie. Brain Res 1994, 656, 329-343.

20. Jeffrey M., McGovern G., Goodsir C.M., Brown K.L., Bruce M.E.: Sites of prion protein accumulation in scrapieinfected mouse spleen revealed by immuno-electron microscopy. J Pathol 2000, 191, 323-332.

21. Jeffrey M., McGovern G., Siso S., Gonzalez L.: Cellular and sub-cellular pathology of animal prion diseases: relationship between morphological changes, accumulation of abnormal prion protein and clinical disease. Acta Neuropathol 2011, 121, 113-134.

22. Juling K., Schwarzenbacher H., Frankenberg U., Ziegler U., Groschup M., Williams J.L., Fries R.: Characterization of a 320$\mathrm{kb}$ region containing the HEXA gene on bovine chromosome 10 and analysis of its association with BSE susceptibility. Anim Genet 2008, 39, 400-406.

23. Kupfer L., Hinrichs W., Groschup M.H.: Prion protein misfolding. Curr Mol Med 2009, 9, 826-835.

24. Legname G., Nguyen H.O., Peretz D., Cohen F.E., DeArmond S.J., Prusiner S.B.: Continuum of prion protein structures enciphers a multitude of prion isolate-specified phenotypes. Proc Natl Acad Sci USA 2006, 103, 19105-19110.

25. Liberski P.P., Brown D.R., Sikorska B., Caughey B., Brown P.: Cell death and autophagy in prion diseases (transmissible spongiform encephalopathies). Folia Neuropathol 2008, 46, $1-25$. 
26. Malik I., Turk J., Mancuso D.J., Montier L., Wohltmann M., Wozniak D.F., Schmid, R.E., Gross R.W., Kotzbauer P.T.: Disrupted membrane homeostasis and accumulation of ubiquitinated proteins in a mouse model of infantile neuroaxonal dystrophy caused by PLA2G6 mutations. Am J Pathol 2008, $172,406-416$.

27. Moore R.A., Taubner L.M., Priola S.A.: Prion protein misfolding and disease. Curr Opin Struct Biol 2009, 19, 14-22.

28. Okada H., Iwamaru Y., Fukuda S., Yokoyama T., Mohri S.: Detection of disease-associated prion protein in the optic nerve and the adrenal gland of cattle with bovine spongiform encephalopathy by using highly sensitive immunolabeling procedures. J Histochem Cytochem 2012, 60, 290-300.

29. Paulick M.G., Bertozzi C.R.: The glycosylphosphatidylinositol anchor: a complex membrane-anchoring structure for proteins. Biochemistry 2008, 47, 6991-7000.

30. Piccardo P., Cervenak J., Bu M., Miller L., Asher D.M.: Complex proteinopathy with accumulations of prion protein, hyperphosphorylated tau, alpha-synuclein and ubiquitin in experimental bovine spongiform encephalopathy of monkeys. J Gen Virol 2014, 95, 1612-1618.

31. Raju T.N.: The Nobel chronicles. 1997: Stanley Ben Prusiner (b 1942). Lancet 2000, 356, 260.

32. Rudd P.M., Merry A.H., Wormald M.R., Dwek R.A. Glycosylation and prion protein. Curr Opin Struct Biol 2002, 12, 578-586.

33. Sander P., Hamann H., Drogemuller C., Kashkevich K., Schiebel K., Leeb T.: Bovine prion protein gene (PRNP) promoter polymorphisms modulate PRNP expression and may be responsible for differences in bovine spongiform encephalopathy susceptibility. J Biol Chem 2005, 280, 37408-37414.

34. Santuccione A., Sytnyk V., Leshchyns'ka I., Schachner M.: Prion protein recruits its neuronal receptor NCAM to lipid rafts to activate p59fyn and to enhance neurite outgrowth. J Cell Biol 2005, 169, 341-354.

35. Yuan A.H., Hochschild A.: A bacterial global regulator forms a prion. Science 2017, 355, 198-201. 